\title{
TREATMENT FIDELITY: ITS IMPORTANCE AND REPORTED FREQUENCY IN APHASIA TREATMENT STUDIES
}

\begin{abstract}
Treatment fidelity is a measure of the reliability of the administration of an intervention in a treatment study, and has important linkages and implications for the ultimate implementation of evidence-supported interventions. In this study, we examined aphasia treatment studies published in the last 10 years for the reporting of treatment fidelity. We found that only $10 \%$ of studies over the last 10 years reported treatment fidelity, which is less than other related disciplines. We discuss the means by which we assess fidelity and recommend the consistent reporting of treatment fidelity in all treatment studies.
\end{abstract}

\section{STUDY DESCRIPTION}

Treatment fidelity refers to how well a treatment condition was implemented as planned in a research study (Vermilyea, Barlow, \& O'Brien, 1984; Yeaton \& Sechrest, 1981), and includes an assurance of reliability that the treatment conditions being compared are sufficiently different from each other (Moncher \& Prins, 1991).

Reported treatment fidelity is a critical component to the ultimate implementation of any evidence-supported intervention. Firstly, we must be certain that a treatment that may ultimately become an evidence-based practice has been consistently administered throughout a clinical trial or treatment study, to ensure that the conclusions of the study are valid. The phenomenon of "therapist drift" has been observed in which clinicians, even those who are participating in a treatment study, may make small but critical changes to the administration of a treatment in response to client performance. This can be done unintentionally or even unknowingly, but may be part of a clinician's expertise in matching client performance to treatment task. Thus, it is important to actively assess the reliability with which a studied treatment was administered in a study. When treatment integrity is not measured within a treatment study, results might be attributable to the planned treatment rather than the actually implemented treatment. Thus, reports of treatment integrity potentially affect the internal validity of a treatment study.

Secondly, once a treatment is supported by evidence, practitioners will need to understand and be able to implement the critical components of the treatment in real settings. A critical bridge between the accumulated evidence for a treatment and its implementation in real practice is an understanding of its critical components, which typically begins with the establishment of and the measure with which fidelity has been assessed (Fixsen, Naoom, Blasé, Friedman, \& Wallace, 2005; Frances, Sweeney, \& Clarkson, 1985). This is critical for practitioners who may need to make adjustments to the administration of any particular treatment in order to accommodate client values, logistics, and institutional policies.

Finally, as we envision the use of evidence-based practices in typical clinical settings, we will need fidelity measures with which quality assurance teams can determine how well and how effectively clinicians are administering a given evidence-based intervention. 
In the last few years, a number of other disciplines have begun to self-examine their use of fidelity measures as practices from schools, health care, psychology, and medicine strive to implement evidence-based practices (Sanetti, Gritter, \& Dobey, 2011;

The purpose of this paper is to review published treatment studies in the journal Aphasiology for their inclusion of reported treatment integrity measures, and to discuss methods by which reported treatment fidelity can be improved.

\section{METHODS}

Studies published in Aphasiology from the last ten years (2002-2011) were reviewed. The following criteria were set for publication selection. Studies need to be an empirical study of an intervention administered across multiple sessions, and self-identified as a "treatment study". Publications that were reviews of previously work, re-publications of older studies (e.g., "CAC Classics"), and retrospective studies were excluded. A total of 134 studies met these inclusion and exclusion criteria and were entered into the review.

Each of these 134 studies was reviewed for identifying information, general description of the study design, dependent and independent variables, indication of whether any measure of treatment fidelity was explicitly included. The treatment description in each study was judged as to whether level of detail was sufficient for replication.

The study design for each study was categorized based on groupings used by Gresham and colleagues (Gresham, Gansle, Noell, Cohen, \& Rosenblum, 1993; see Table). Dependent and independent variables were listed in terms used by the study author(s).

Studies were also coded as to whether the treatment description was operationally described at a level sufficient for replication. Raters were asked to consider "Could you replicate/implement this treatment based on the description in this publication?" If sufficient description was offered to allow for implementation, than "Yes" was coded. If the study was using a treatment for which there were additional published references or resources, then "Yes" was coded.

Binary (yes/no) coding was also used to indicate whether studies reported any measure of treatment fidelity. Measures such as observations of treatment adherence, use of a training manual, or measures of procedural reliability were coded as "Yes". For those studies that indicated treatment fidelity, additional details were recorded about how treatment integrity was established, sources used for treatment integrity, and how implementers of the treatment were trained.

\section{RESULTS}

The average number of treatment studies appearing in each of the five years was 12.8 studies appearing/year. Overall, the mean number of participants per study was 2 (range $=1-20$ participants). 
The majority of appearing studies incorporated a multiple baseline design, followed by other single subject designs.

$28 \%$ of the studies were judged to provide insufficient treatment description to allow for replication based on the publication alone. Some studies described fairly complex treatments that lacked detail; others described treatments that required clinician decision-making regarding tasks and trials, and criteria for these decisions were not offered.

Thirteen of the 134 studies (10\%) explicitly reported some aspect of treatment fidelity. The majority of these 13 studies checked adherence to steps in the treatment protocol by having one or more raters review videotapes from a sample (10-20\%) of the training sessions, and asking the raters to indicate whether each step was observed. In these seven studies, a percentage of the treatment steps completed in the sampled sessions was reported. When more than one rater was used for checking protocol steps in sampled sessions, point-to-point agreement between the raters was reported. The Figure shows the total number of treatment studies per year with the number of studies reporting treatment fidelity.

Two of these studies that reported treatment fidelity described the use of a training manual. In one other study, implementers were trained prior to initiation of the treatment study via role playing (Melton \& Bourgeois, 2005). So, three of the reviewed studies explicitly mentioned how implementers of the treatment were themselves trained. One study (Hickey, Bourgeois, \& Olswang, 2004) reported both a training manual and independent ratings of training adherence.

\section{DISCUSSION}

The purpose of this review was to describe the reporting of treatment fidelity among treatment studies published in Aphasiology in the last ten years. Of 134 reviewed studies, approximately $10 \%$ reported some measure of treatment fidelity. The primary method used in these studies was review of video samples of treatment sessions for evaluation of adherence to treatment protocol steps.

The percentage of studies reporting measures of treatment integrity in this review was similar to reviews of treatment integrity in the school-based intervention literature (Peterson, Homer, \& Wonderlich, 1982; Gresham, Gansle, Noell, Cohen, \& Rosenblum, 1993) but generally less that more recent evaluations of reported treatment fidelity in other disciplines (McEvoy, Shores, Wehby, Johnson, \& Fox, J J., 1990; Sanetti, Gritter, \& Dobey, 2011). Furthermore, some areas in speech-language pathology are paying particular attention to this issue (Wheeler, Baggett, Fox, \& Blevins, 2006; Schlosser, 2002).

Additional discussion will focus on methods of conducting treatment integrity. Attending to treatment integrity in our single studies will make our conclusion more reliable and robust and set the stage for the implementation of evidence-based practice. 


\section{REFERENCES}

Cochrane, W. S. \& Laux, J. M. (2008). A survey investigating school psychologists' measurement of treatment integrity in school-based interventions and their beliefs about its importance. Psychology in the Schools, 45, 499-507.

Fixsen, D. L., Naoom, S. F., Blasé, K. A., Friedman, R. M., \& Wallace, F. (2005). Implementation research: A synthesis of the literature. Tampa, FL: University of South Florida, Louis de la Parte Florida Mental Health Institute, The National Implementation Research Network (FMHI Publication \#231).

Frances, A., Sweeney, J. \& Clarkin, J. (1985). Do psychotherapies have specific effects? American Journal of Psychotherapy, 39, 159-174.

Gresham, F. M., Gansle, K. A., Noell, G. H., Cohen, S \& Rosenblum, S. (1993). Treatment integrity of school-based behavioral intervention studies: 1980-1990. School Psychology Review, 22, 254-273

McEvoy, M. A., Shores, R. E., Wehby, J. H., Johnson, S. M. \& Fox, J J. (1990). Special education teachers' implementation of procedures to promote social interaction: Reported treatment fidelity among children in integrated settings. Education and Training in Mental Retardation, 25, 267-276.

Moncher, F. J. \& Prinz, R. J. (1991). Treatment fidelity in outcome studies. Clinical Psychology Review, 11, 247-266.

Noell, G. H., Gresham, F. M., \& Gansle, K. A. (2002). Does treatment integrity matter? A preliminary investigation of instructional implementation and mathematics performance. Journal of Behavioral Education, 11, 51-67.

Sanetti, L. M. H., Gritter, K. L., \& Dobey, L. M. (2011). Treatment integrity of school interventions with children in the school psychology literature from 1995 to 2008. School Psychology Review, 40, 72-84.

Schlosser, R. W. (2002). On the importance of being earnest about treatment integrity. AAC Augmentative and Alternative Communication, 18, 36-44.

Peterson, L., Homer, A. L. \& Wonderlich, S. A. (1982). The integrity of independent variables in behavior analysis. Journal of Applied Behavior Analysis, 15, 477-492.

Vermilyea, B. B., Barlow, D. H. \& O’Brien, G. T. (1984). The importance of assessing treatment integrity: An example in the anxiety disorders. Journal of Behavioral Assessment, 6, 111.

Wheeler, J. J., Baggett, B. A., Fox, J., \& Blevins, L. (2006). Treatment integrity: A review of intervention studies conducted with children with autism. Focus on Autism and Other Developmental Disabilities, 21, 45-54.

Yeaton, W. H. \& Sechrest, L. (1981). Critical dimensions in the choice and maintenance of successful treatment: strength, integrity, and effectiveness. Journal of Consulting and Clinical Psychology, 49, 156-167. 

Table. Study design coding categories used in the review (from Gresham, Gansle, Noell, Cohen, \& Rosenblum, 1993).

Group = use of an experimental group and a control group or a comparison of two or more treatment groups

Withdrawal designs = comparisons within subject designs in which changes are compared across phases of the study (e.g., A/B/A/B, etc.)

Multiple Baseline designs = Comparisons of both within and between subjects and/or behaviors

Alternating Treatments $=$ Alternating treatment components across sessions or days

Changing Criterion designs $=$ Within series change strategies in which the dependent variables is brought under the control of established and shifting criteria 
Figure. The total number of aphasia treatment studies published each year with the number of those studies reporting treatment fidelity measures.

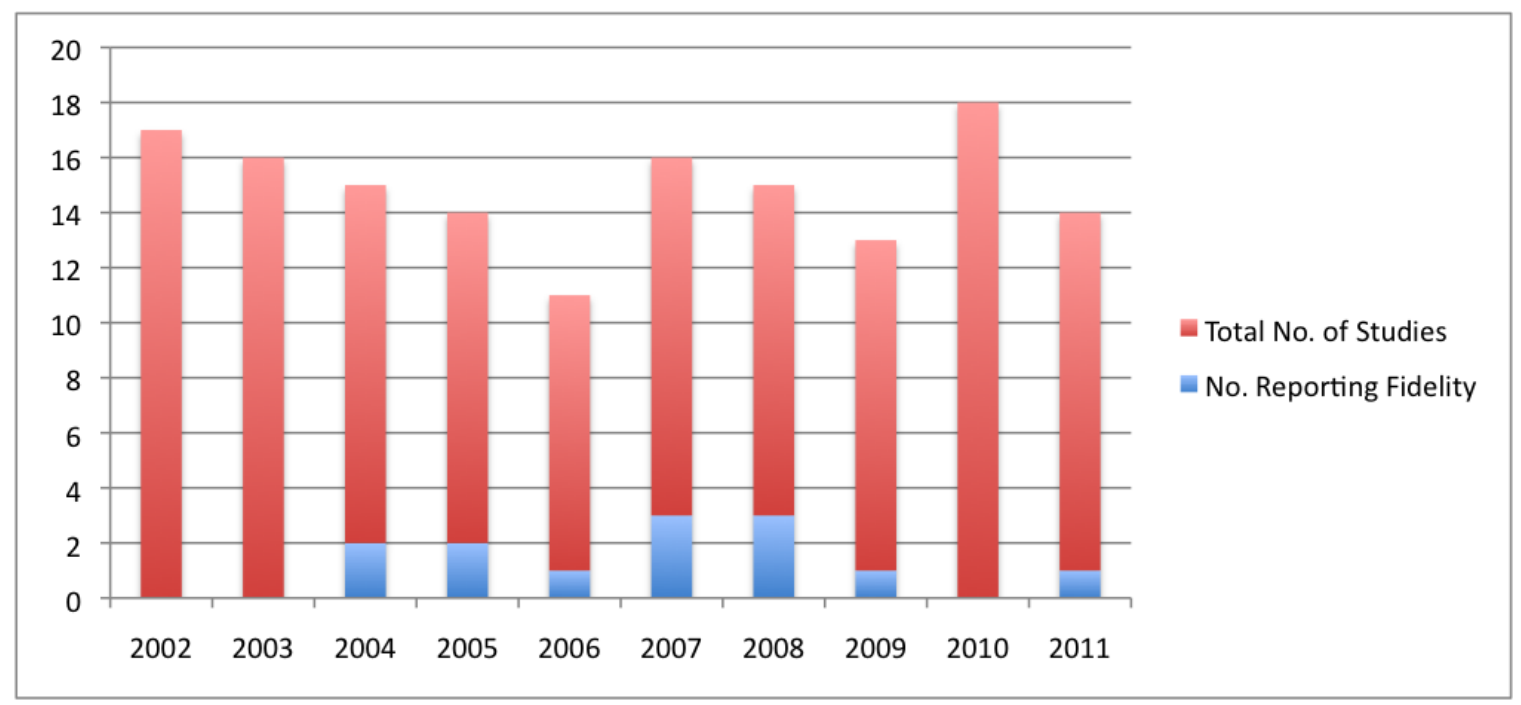

